ChAPTER 429

\title{
Capability Test for a Digital Cognitive Flight Crew Model
}

\author{
Andreas Lüdtke ${ }^{1}$,Jan-Patrick Osterloh ${ }^{1}$, Tina Mioch ${ }^{2}$, Joris Janssen $^{2}$ \\ ${ }^{1}$ OFFIS e.V., Escherweg 2, 26127 Oldenburg, GERMANY \\ ${ }^{2}$ TNO Human Factors, Kampweg 5, 3769 DE Soesterberg, \\ THE NETHERLANDS
}

\begin{abstract}
The objective of the HUMAN project is to develop virtual test pilots for cockpit systems, in order to improve the human error analysis during cockpit system design. Virtual test pilots should supplement simulator-based testing of new cockpit systems with human pilots, by providing the possibility to simulate human behavior in early design phases. In this paper we will present how we modeled and tested four relevant basic capabilities, necessary for the simulation of pilot behavior, namely crew coordination, sophisticated perception, reactive behavior and multitasking.
\end{abstract}

Keywords: Cognitive flight crew model, cognitive architecture, rule-based modeling

\section{INTRODUCTION}

In the project HUMAN (funded by the European Commission under the 7th Framework Programme) a digital cognitive pilot crew model is developed which will be used as a virtual tester of complex cockpit system designs in realistic flight scenarios. The paper describes our modeling approach, and shows that the model is able to simulate some relevant basic capabilities to cope with highly dynamic flight 
scenarios. To demonstrate the capabilities our model has been connected to a generic cockpit simulator within a simulated air traffic environment, called simulation platform throughout the paper. We simulated flight scenarios in which the model has to interact with an Advanced Human Machine Interface (AHMI) in order to manipulate the Advanced Flight Management System (AFMS; both developed by the German Aerospace Center, Braunschweig, Germany) and further cockpit systems (e.g. the autopilot) to navigate the aircraft according to flight procedures. The basic capabilities investigated in this paper are: (1) Crew Coordination, to simulate a flight crew (sharing of task execution and communication); (2) Perception, to simulate sophisticated perception in terms of a restricted visual field with a visual focus including attention according to current visual focus and realistic times for eye-movements; (3) Reactive Behavior, to react to events (e.g. ATC calls, warning lights) by initiating adequate flight procedures (e.g. an altitude change maneuver); (4) Multitasking, in terms of maintaining multiple goals at the same time and switching between them, as well as interrupting a goal to deal with reactive goals.

These four capabilities are achieved with the cognitive architecture CASCaS (Cognitive Architecture for Safety Critical Task Simulation), whose key concept is the theory of behavior levels (cf. Anderson (2000) or Rasmussen (1983)). A flight crew is modeled by using two instances of the model: a pilot flying (PF) model and a pilot monitoring (PM) model. To test the model capabilities we devised and simulated two flight scenarios and defined expected plausible behavior. The resulting model behavior has then been tested against these expectations.

The following section presents more details on the four basic capabilities and describes the modeling approaches within the cognitive architecture CASCaS. Afterward the process for testing the capabilities and the test results are described.

\section{MODELLING THE BASIC CAPABILITIES WITHIN A COGNITIVE ARCHITECTURE}

The first basic capability we modeled is to simulate a flight crew. The crew is a very important safety net and consists of two pilots, a pilot flying (PF) and a pilot nonflying (PNF). While the PF has control of the aircraft, the PNF monitors the actions of the PF and the aircraft, and has other duties like communication with the air traffic controller (ATC). Main duty of the PNF is monitoring, thus this pilot is therefore referred to as the pilot monitoring (PM) in this paper. Monitoring the PFs actions includes sharing of task execution, e.g. procedures that foresee cross-checks at certain points. This implies communication between PF and PM.

The second important basic capability concerns perception. This capability is needed to simulate realistic visual attention allocation with regard to what a pilot can perceive at a certain point in time of the simulation. It is based on visual fixations and transitions between these fixations (saccades). Therefore it was necessary to create a restricted visual field with a visual focus and modeling realistic times for eye-movements. 
[Type text]

The third basic capability of our model is to react to events. These events can be for example ATC calls (clearances for altitude) and warning lights. When perceiving an event, the model should initiate the associated flight procedure (e.g. an altitude change procedure).

The fourth and final basic capability which has been modeled is to simulate multitasking. The model is capable of maintaining multiple goals at the same time, switching between them, as well as interrupting a goal to deal with reactive goals.

The basic capabilities have been modeled within the cognitive architecture CASCaS, which was developed by OFFIS in the EU project ISAAC (see Lüdtke et al. (2006) and Lüdtke et al. (2009) for further information on CASCaS). The architecture is based on a flight procedure formalization in the form of "if-then" rules. The rules formally describe a mental representation of flight procedures. In order to perform a simulation, the flight procedure rules are uploaded to the cognitive architecture. A cognitive architecture with uploaded procedure rules is what we call a pilot model. The cognitive architecture can be understood as an interpreter or executor of formal flight procedure rules. Within a simulation platform the pilot model interacts with a system under investigation (e.g. the AHMI/AFMS) and a simulated environment (including the aircraft). A simulation kernel synchronizes the different models and organizes the dataflow.

CASCaS is based on Rasmussen's (1983) three behavior levels in which cognitive processing takes place: skill-based, rule-based and knowledge-based behavior. The levels of processing differ with regard to their demands on attention control dependent on prior experience: skill-based behavior is acting without thinking in daily operations, rule-based behavior is selecting stored plans in familiar situations, and knowledge-based behavior is coming up with new plans in unfamiliar situations. Anderson (2000) distinguishes very similar levels, but uses the terminology of autonomous, associative, and cognitive level, which will be used throughout the paper. In HUMAN CASCaS has been adapted to integrate the basic capabilities. The modeling effort focuses on the associative and cognitive behavior level. Figure 1 gives an overview on the components of CASCaS. These components form the following control loop: The "Perception" component retrieves the current situation from the "Simulation Environment", and stores the information in the "Memory" component. The "Processing" component contains components for the behavior layers. These layers can retrieve information from the memory and process this information according to their cognitive cycle (rule-based or knowledge-based). The layers may store new information in the memory, or start motor actions in the "Motor" component. 


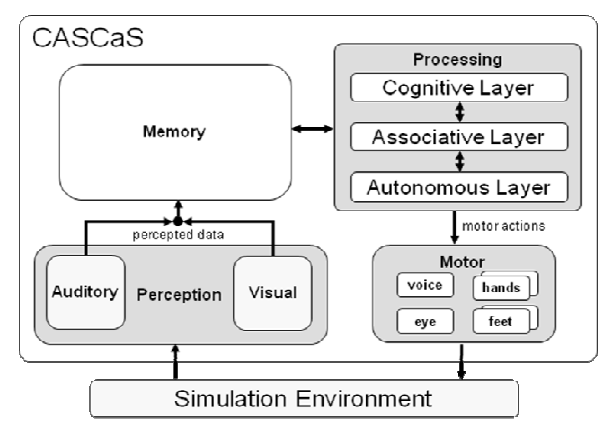

Figure 1. CASCaS Architecture

\section{CREW COORDinAtion}

In order to simulate a crew, two instances of CASCaS are running in parallel. Each instance has its own procedure set, one for the PF and one for the PM. In addition, CASCaS has been extended with two new components, a vocal motor and an auditory percept component. The rule-based language has been extended with vocal actions. A vocal action consists of a text that is spoken, a variable instantiation which represents the meaning of the text and a receiver. See Figure 2 for an example of a rule with a vocal action. The time for the vocal action is determined by the text "confirm target altitude", estimated with 190 words/min. The vocal action is transferred to its receiver, in this example the modeled Pilot Monitoring (PM), via the simulation platform. The receiver perceives the text and stores the instantiated variable ("cross_check_altitude_request $=1$ ") in the memory component. These memory entries can then trigger reactive rules, see the section on "Reactive Behavior" below.

\begin{tabular}{ll} 
Rule 103005, type=regular & rule header \\
Goal (al_ap_change_altitude_cc) & $\begin{array}{l}\text { active goal } \\
\text { condition }\end{array}$ \\
Condition (target_altitude == fcu_altitude) & \\
$\rightarrow$ & spoken text \\
Vocal ('confirm target altitude', & $\begin{array}{l}\text { instantiated variable } \rightarrow \text { memory } \\
\text { entry for receiver } \\
\text { receiver } \\
\text { cross_check_altitude_request=1, }\end{array}$ \\
soal (al_ap_change_altitude_cc_wait) & suboal \\
\hline
\end{tabular}

Figure 2. Rule activating a vocal action

The vocal action allows the two instances of CASCaS to interact with each other verbally.

\section{PerCeption}

In order to model eye-movements and a visual field, information on the topology of the environment is needed. For example, information is needed about the locations 
[Type text]

of instruments, their size and colors. We defined an XML schema that allows us to describe the instruments in terms of areas of interest (AOI), together with several parameters, like location and size. This is a new input for the percept component of CASCaS. Based on this information, eye- and head movement times can be calculated. We use a 2 degree angle of focus, and a visual field angle of 170 degree horizontal and 110 degree vertical. The visual field is used in order to model selective attention (also called bottom-up attention). Selective attention refers to the phenomenon of automatic shifts of attention due to external events, like a flashing signal, or a moving object. A more detailed description on the percept component can be found in (Osterloh \& Lüdtke 2008).

\section{REACTIVE BEHAVIOR}

There are two types of reactivity: The first type is a non-cognitive, almost automatic reaction to an event. In this case, no reasoning needs to be done, since there is a standard procedure to handle the event. A reactive rule on the associative layer is activated. Reactive rules have the same structure and semantic like regular rules (cf. Figure 2), with the exception that they are not associated to a goal (on the left-hand side of the rule). As soon as the condition of a reactive rule evaluates to true, the rule is fired, independently from the current active goals.

The second type of reactivity is the reaction to events that involve the cognitive layer, e.g. if a decision needs to be made whether a standard procedure is appropriate at a particular moment. In that case, a goal to handle the event becomes active on the cognitive layer, which reasons about appropriate reaction. The cognitive layer may decide to activate a goal on the associative layer if standard procedures are appropriate, or decide to handle the event itself.

\section{MULTITASKING}

Multitasking is modeled in three ways in CASCaS. First, on the associative layer, several goals can be interleaved. The mechanisms for interleaving are based on the general executive for multitasking of Salvucci (2005). Furthermore, the reactive capabilities of the associative layer, which are described in the previous section, allow interrupting an ongoing goal by adding a new goal onto the agenda that is processed immediately.

Second, goals on the cognitive layer are executed based on their priority. It is possible that a currently executed goal is stopped if another goal has a higher priority. However, the first goal is not automatically resumed after finishing the second goal; it is first evaluated whether the first goal is still relevant to the situation by checking its activation conditions again.

A third way of multitasking is realized because the associative and cognitive layer run in parallel and interact in the following ways: (1) The cognitive layer can start (and thus delegate), monitor, temporarily halt, resume and stop activities on the associative layer by manipulating the associative layer's goal agenda. Monitoring of the associative layer is realized through determining whether the appropriate goals 
are placed in the goal agenda. (2) The associative layer can inform the cognitive layer about the status of rule execution. For example, the current execution is stuck because no rules are available in long-term memory for the chosen goal or execution of a perceived event cannot be started for the very same reason. In these cases the cognitive layer starts to perform the goal or event. (3) The cognitive layer can take over control at any time. Currently this is initiated by setting the parameter "Consciousness". If the value is "associative" then every event will first be processed if possible and the cognitive layer becomes only active if no rules are available. If the value is "cognitive" then the cognitive layer processes each event independent of the availability of rules (more details can be found in Lüdtke et al. 2009).

\section{CAPABILITY TEST}

We devised and simulated two main scenarios in order to test the basic capabilities. For each scenario expected plausible behavior has been defined, and resulting model behavior has then been tested against these expectations. The plausibility of the model behavior has been analyzed based on time series data recorded during the virtual simulation. The results show that the model is able to perform basic behavior in flight scenarios in a way as it is expected based on human pilot behavior.

\section{SCENARIO DESIGN}

In order to prove the basic capabilities, two scenarios have been designed, in which the basic capabilities are needed to fly the aircraft safely. The basis for all scenarios is the same flight plan on flight level 90 (9000 feet) at 200 knots. The flight is controlled via a scenario controller, which induces events, like ATC requests, based on the current position of the aircraft.

\section{SCENARIO 1}

The first scenario tests three capabilities: crew coordination, multitasking and reactive behavior. The aircraft is flying in cruise mode on flight level 90 . The basic duty of the PF and the PM is (under normal conditions) to monitor the flight. In the scenario, an ATC call requests the pilots to change the altitude. The crew has to react to this event (reactive behavior), has to interrupt the monitoring task (multitasking), and then it has to follow the procedure for an altitude change via autopilot, which includes cross-checks (crew coordination). When the altitude is changed, both pilots have to resume their monitoring task (multitasking). In order to change the altitude, the PF has to dial the new flight level into the autopilot using the altitude selector. After the PF has selected the new altitude the PM should compare the flight level cleared by the ATC with the flight level dialed in by the PF. The PM and PF models should behave in the following way: 
[Type text]

1. If the value dialed by the PF is correct, the PM should acknowledge verbally and the PF should answer the acknowledgement verbally and activate the new target altitude by pulling the altitude selector.

2. If the value dialed by the PF in incorrect, PM should reject which should trigger the PF to dial in the correct target altitude. The PM should check again and acknowledge verbally. PF should answer the acknowledgement verbally and activate the new target altitude.

In the scenario the second case can be forced by the scenario controller by overwriting the value of the altitude selector. Figure 3 shows the behavior for the first alternative.

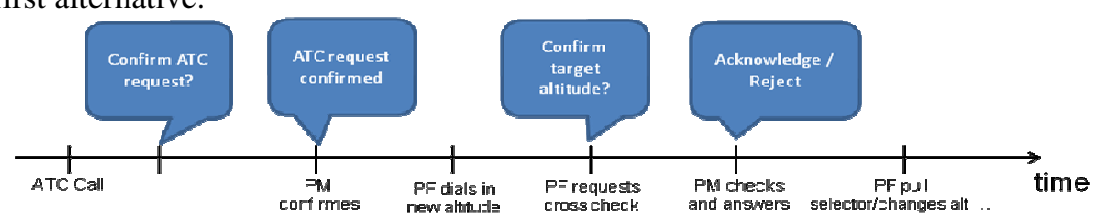

Figure 3. Expected behavior for correct crew coordination

\section{SCENARIO 2}

The second scenario is dedicated to test the basic capability "Perception". This mainly concerns the test of the visual component, particularly the focus and the visual field. The scenario has been divided into three steps:

1) The PF model has to perform procedures that involve reading autopilot flight mode annunciations on the Primary Flight Display (PFD) and values from the engine display. In order to read the values the model has to simulate eyemovements.

2) While the visual focus is on the engine display, a flight mode is changed by the scenario controller. This is displayed on the PFD with a flashing box for 10 seconds around the flight mode annunciation. The flashing box should attract the visual attention of the PF model, because it appears in the visual field. If the PF model detects the mode change, a vocal output should be generated.

3) Finally, the PF should shift the visual focus to the track ball. While the PF focuses the trackball, the scenario controller changes the flight mode. This time the PFD and thus the mode change is outside the visual field, because the trackball is located near the knees of the pilot. In this case the mode change should not be detected.

\section{RESULTS OF THE CAPABILITY TEST}

\section{Scenario 1}

As described above, scenario 1 tests the basic capabilities crew coordination, multitasking, and reactive behavior.

Crew coordination: As can be seen in Figure 4, for the cross-check, the two 
cognitive models communicate with each other, the light gray bar representing the $\mathrm{PF}$, the dark gray one representing the PM. It can also be seen that the communication depends on the status of the environment. The PF's actions depend on the vocal input he receives from the PM. In Figure 4, the dialed-in altitude is accepted by the PM, and thus executed by the PF, whereas in Figure 5, the dialed-in altitude is not accepted, and therefore the PF needs to correct the altitude.

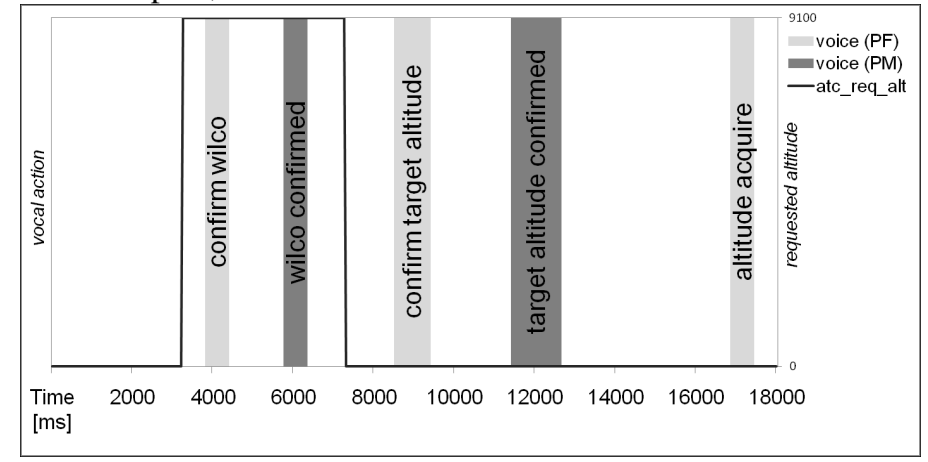

Figure 4. Diagram depicting verbal protocols from PF and PM, taken from logfile. The flight level dialed by the PF corresponds to the flight level requested by ATC.

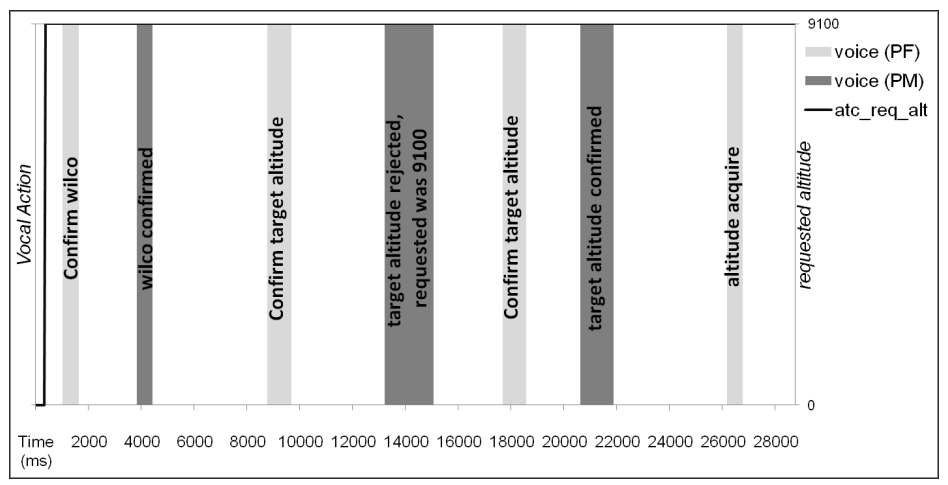

Figure 5. Diagram depicting verbal protocols from PF and PM, taken from logfile. The flight level dialed by the PF does not correspond to the flight level requested by ATC.

Multitasking: As can be seen in Figure 6, the monitoring task is interrupted when the ATC call occurs (at time 82300). After handling the ATC call (around time 112300), the monitoring task is resumed at the same point as it was interrupted before.

Reactive behavior: When receiving the ATC call, the monitoring task is interrupted and it is reacted to the ATC call by choosing the appropriate procedure to handle the ATC call (i.e. change altitude). 


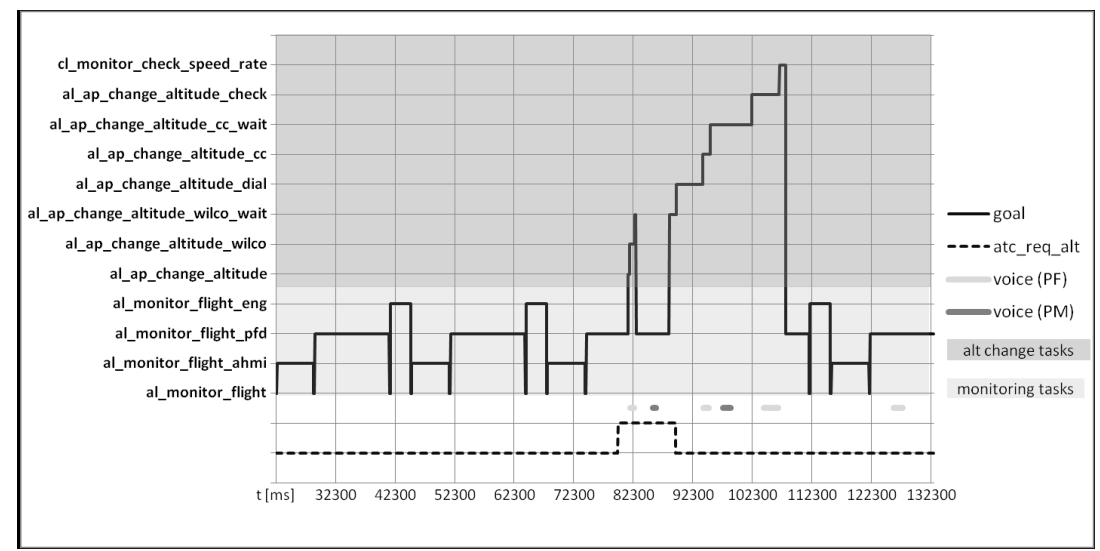

Figure 6. Diagram depicting switching between monitoring tasks and altitude change task

\section{SCENARIO 2}

Scenario 2 tests the perception, particularly the focus and the visual field. As can be seen in Figure 7, the percept component is able to focus on different parts of the interface.. The first mode change is recognized by the pilot, as it is in the visual field. The second mode change, even though the same event occurs (flashing box on screen), is not perceived by the PF, as his eyes are on the mouse, which means that the flight mode annunciation is outside the visual field. As can be seen, no verbal action is taken by the pilot.

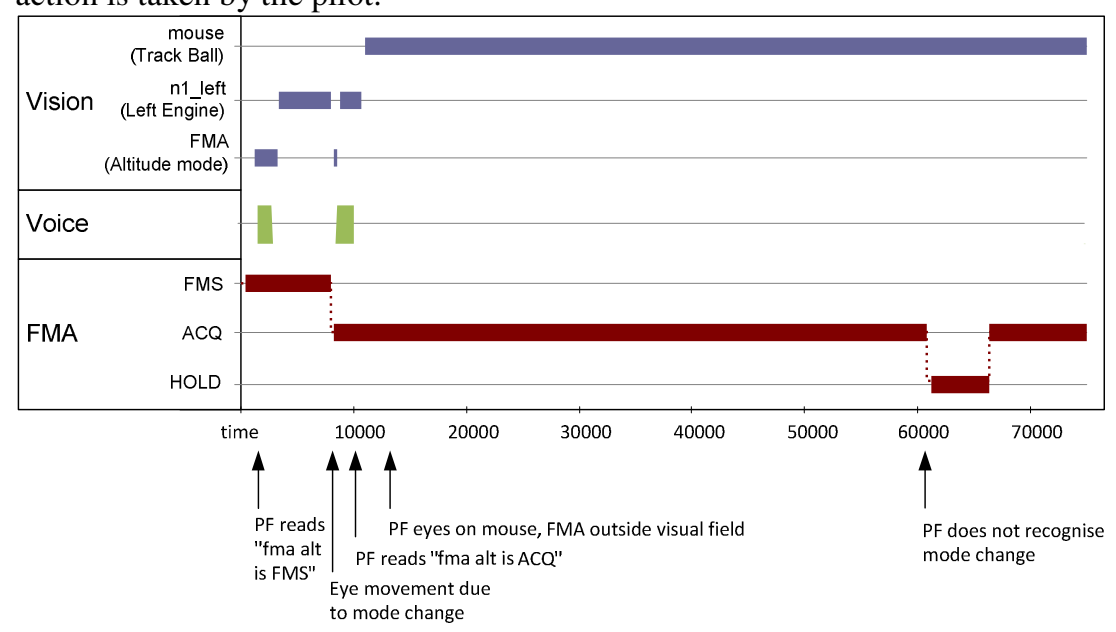

Figure 7. Diagram that displays the location of the eye gaze, and recognition of events in the visual field. 


\section{CONCLUSION AND NEXT STEPS}

In this paper, we presented how we modeled basic capabilities, relevant for simulation of pilot behavior. These basic capabilities where tested within two scenarios against expected plausible behavior. The next validation step will be to compare the model data with human pilot data.

It has to be pointed out that our model abstracts from several features of human behavior. Especially, we have not modeled the influence of performance shaping factors like fatigue or emotions. For example it is well known that under fatigue, pilot performance degrades significantly. Our model represents pilots that are impacted neither by fatigue nor emotions, thus generated predictions have to be interpreted taking this into account.

The work described in this paper is funded by the European Commission in the $7^{\text {th }}$ Framework Programme, Transportation under the number FP7 -211988.

\section{REFERENCES}

Anderson, J. R. (2000), Learning and Memory, John Wiley \& Sons, Inc.

Anderson, J. R., Bothell, D., Byrne, M. D., Douglass, S., Lebiere, C. \& Qin, Y. (2004). An Integrated Theory of the Mind. Carnegie Mellon University, Pittsburgh and Rice University, Houston.

Lüdtke, A., Cavallo, A., Christophe, L., Cifaldi, M., Fabbri, M., Javaux, D.: Human Error Analysis based on a Cognitive Architecture. In: Reuzeau, F., Corker, K., Boy, G. (eds) Proceedings of HCI-Aero, pp. 40-47, Cépaduès-Editions, France (2006)

Lüdtke, A., Osterloh, J.-P., Mioch, T., Rister, F., Looije, R. Cognitive Modelling of Pilot Errors and Error Recovery in Flight Management Tasks. Proceedings of the 7th Int. Working Conference on Human Error, Safety, and System Development, Brussels, Belgium, Sept. 23-25, 2009

Rasmussen, J. (1983), "Skills, Rules, Knowledge: Signals, Signs and Symbols and other Distinctions in Human Performance Models" IEEE Transactions: Systems, Man and Cybernetics, SMC-13, pp.257-267

Salvucci, D. D. (2005). A multitasking general executive for compound continuous tasks. Cognitive Science 29, S. 457-492. 